\title{
Endovascular Embolization of Pseudoaneurysms Complicating Pancreatitis Using Microcoils: Case Series
}

\author{
Kishor Taori ${ }^{1}$, Jawahar Rathod ${ }^{1}$, Amit Disawal ${ }^{1}$, Rajesh Mundhada ${ }^{2}$, Atul Rewatkar ${ }^{2}$, \\ Vishal Bakare $^{1}$, Prasad Wavare ${ }^{1}$, Rakhi P. Puria ${ }^{1}$ \\ ${ }^{1}$ Department of Radio-Diagnosis, Government Medical College and Hospital, Nagpur, India \\ ${ }^{2}$ Orange City Hospital, Nagpur, India \\ Email: kishortaori@gmail.com
}

Received December 6, 2012; revised January 5, 2013; accepted January 13, 2013

\begin{abstract}
Purpose: To determine whether endovascular embolization of pseudoaneurysms complicating pancreatitis with microcoils was associated with better therapeutic outcome. Materials and Method: The prospective clinical study was carried out in the Department of Interventional Radiology for a period of 2 years extending from May 2010 upto August 2012 on 16 male patients in the age group of about 30 - 55 years, with each one of them having vascular complications as a sequel to pancreatitis, after obtaining well informed written consent from each one of them. Results: The outcome of the procedure was judged by the following parameters-a) Restoration of the blood pressure of the patient; b) Cessation of the hemetemesis and malena; c) Reduction in abdominal pain and discomfort and d) Overall improvement in the general condition of the patient. Each of the patients had been on follow up for at least 6 months, except 2 of them, with no new complaints in any of them. The procedure related mortality was none. Conclusion: Vascular complications of pancreatitis need immediate and accurate diagnosis and prompt treatment. Micro coil embolisation, is a minimally invasive technique which helps to exclude the pseudoaneurysm from the circulation and thus reduces the scope for massive life threatening internal hemorrhage, and is a better alternative to surgery.
\end{abstract}

Keywords: Pancreatitis; Ruptured; Pseudoaneurysms; Visceral Arteries; Microcoils

\section{Introduction}

Pancreatitis is one of the common conditions in patients presenting with acute abdominal pain. Severe pancreatitis occurs in $20 \%-30 \%$ of all patients with acute pancreatitis and is characterized by a protracted clinical course, multiorgan failure, and pancreatic necrosis. Of the various complications of pancreatitis, vascular complications are one of the most dangerous life threatening ones. Vascular complications of acute pancreatitis include thrombosis, erosion of the vessel wall, and pseudoaneurysm formation [1]. Pseudoaneurysms are relatively uncommon complications of acute pancreatitis that occur in approximately $10 \%$ of cases. The wall of the vessel is eroded by released and activated pancreatic enzymes. Pseudoaneurysms most commonly affect the splenic artery, gastroduodenal, pancreaticoduodenal, gastric, and hepatic arteries (in descending order of frequency) [1,2]. Rupture of a pseudoaneurysm may result in fatal haemorrhage into the gastrointestinal tract or other adjacent anatomic structures. Fatal bleeding may be preceded by self-limiting episodes of "herald bleeding". Hence, prompt diagnosis and management of these lesions is necessary to prevent further catastrophe. Here, we report a study of 16 patients with complicating pancreatitis who were successfully embolized using microcoils.

\section{Case Series}

\subsection{Methods and Material}

\section{Patients}

The prospective clinical study was carried out in the Department of Interventional Radiology for a period of 2 years extending from May 2010 upto August 2012. It included total 16 male patients in the age group of about 30 - 55 years, with each one of them having vascular complications as a sequel to pancreatitis, and haven taken prior well informed written consent from each of them. The materials included routine angiography instruments like Gelco, Angiosheath, Terumo guide wires, Head hunter and Cobra catheters, Micro-catheters, Contrast agent and Embolization microcoils of varying sizes and the $\mathrm{C}$ arm machine.

The Inclusion criteria were:

- Patients with pancreatitis having complaints of repeated episodes of severe abdominal pain;

- Hemetemesis and Malena; 
- Falling blood pressure.

The Exclusion criteria were:

- Patients who were unable to lie supine for long time;

- Patients with severe hemodynamic instability.

\subsection{Observations}

Total 16 male patients were included in the study. All were diagnosed to have pancreatitis with vascular complications in the form of pseudoaneurysm formation of varying sizes, and involving different branches of the celiac trunk and superior mesenteric artery as per individual case. The initial diagnosis was made with the help of plain and contrast enhanced CT scan and then conventional angiography was done for further diagnostic and therapeutic purpose. The table (Table 1) showing the distribution of the number of individual cases and site of pseudoaneurysm formation highlights that the gastroduodenal artery was most commonly affected in 6 patients, followed by the hepatic and splenic arteries in 4 \& 3 cases respectively. The table (Table 2) shows the individual case details in the form of the arterial site of involvement, the size and number of pseudoaneurysms, and the number of microcoils used in each case.

\subsubsection{Embolization Technique}

Initially through the right femoral arterial puncture, with help of $4 \mathrm{~F}$ Cobra catheter and guide wire assess was made inside the abdominal aorta. Then with cannulation of the celiac and superior mesenteric arteries was done as per the need. Initial check angiograms were taken to locate the site and number of the pseudoaneurysms. The check angiograms were carefully assessed for the location, size, number, neck size and rupture if present of the pseudoaneurysms and then the appropriate sizes and number of the microcoils were decided. Through the same route, microcoils of varying sizes as per the need were inserted carefully under DSA control to exclude the pseudo aneurysm from circulation. More than $3 \mathrm{mi}-$ crocoils were used in each case for the "sandwich technique" of coil embolization. In one of the cases of splenic artery pseudoaneurysm, all the inserted microcoils mi-

Table 1. Number of individual cases.

\begin{tabular}{cc}
\hline PSEUDO ANEURYSM OF & NUMBER OF CASES \\
\hline Gastro-duodenal artery (GDA) & 6 \\
Hepatic artery & 4 \\
Spleenic artery & 3 \\
Inferior pancreatico-duodenal artery & 2 \\
Common hepatic artery & 1 \\
TOTAL PATIENTS & $\mathbf{1 6}$ \\
\hline
\end{tabular}

Table 2. Individual case details.

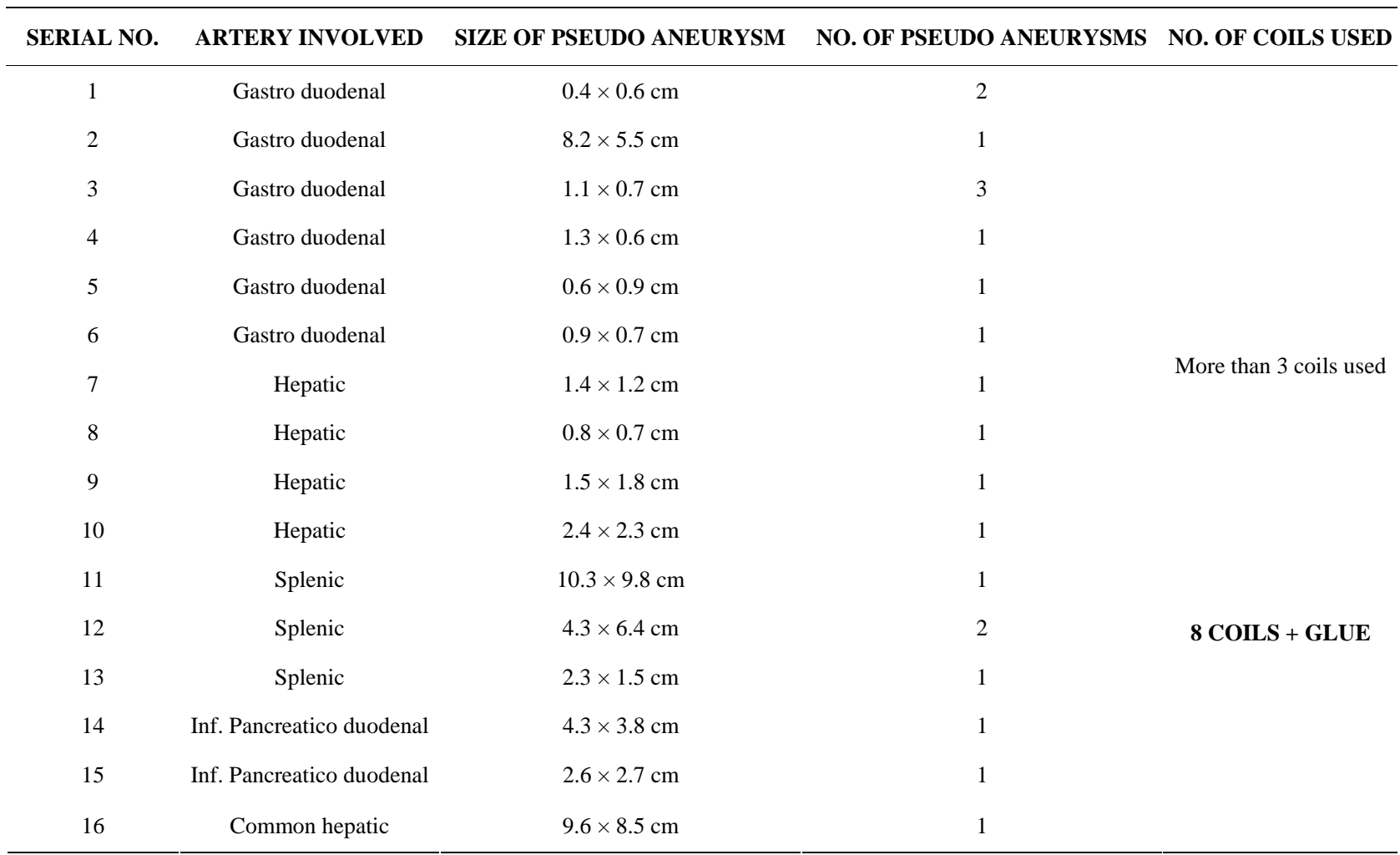


grated distally at acute turn of distal splenic artery. The check angiography showed patent pseudoaneurysm with embolization of the distal splenic artery. Hence, whole of the splenic artery proximal to the microcoils was embolised using Glue (N-butyl Cyanoacrylate) mixed with lipiodol in equal proportion upto the splenic arterial origin. The microcoils prevented the glue from entering into the splenic parenchyma. In each of the cases post embolization check angiogram was taken to assess the success of embolization in the form of exclusion of the pseudoaneurysm from the circulation and non contrast filling of the parent artery (Figures 1(e), 2(c), 3(c), 4(c), 5(c) and 6(c)).

The pseudoaneurysm presented with rupture leading into haemorrhage into the peri-pancreatic collection (Figures 1(a)-(c)) as in the case of hepatic artery pseudoaneurysm, while it presented as hemetemesis and malena due to rupture into the gastrointestinal tract in some cases (Figures 2(a), 2(b) and 6(a)), while some were incidentally detected during scanning. Some of these pseudoaneurysms were relatively smaller in size (Figures 1(c), 1(d) and 2(b)), while in other cases they were larger in size (Figures 3(a), 3(b), 4(a), 4(b) and 5(a)).

\subsubsection{Outcome}

The outcome of the procedure was judged by the following parameters:

1) Restoration of the blood pressure of the patient;

2) Cessation of the hemetemesis and malena;

3) Reduction in abdominal pain and discomfort;

4) Overall improvement in the general condition of the patient.

\subsubsection{Follow Up}

Usually the patients were kept admitted for 5 - 7 days after the procedure to see for any new procedure related complications. There was no such significant event noted, except for post embolization pain which resolved with

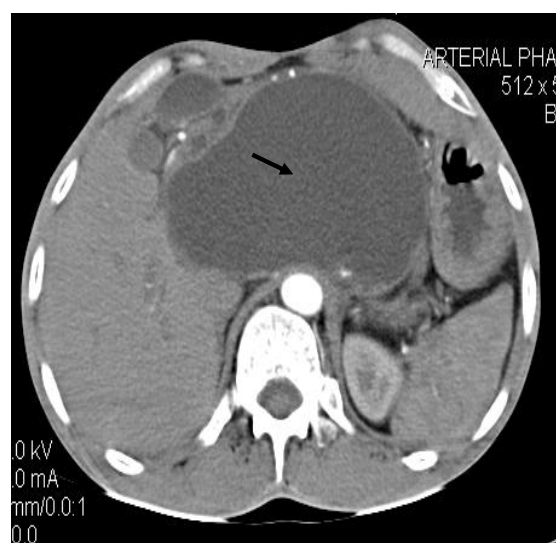

(a)

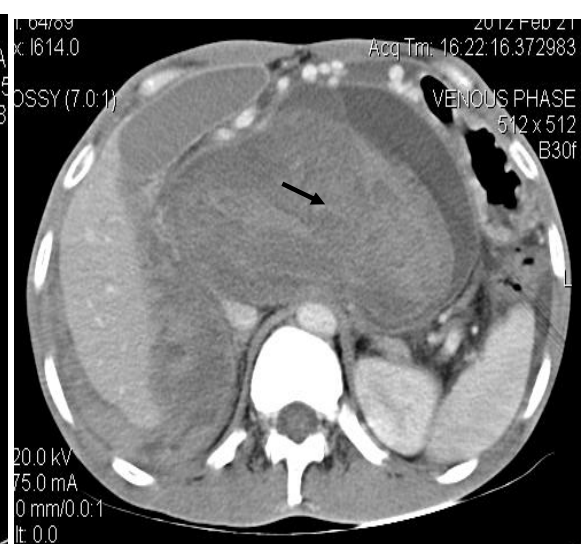

(b)

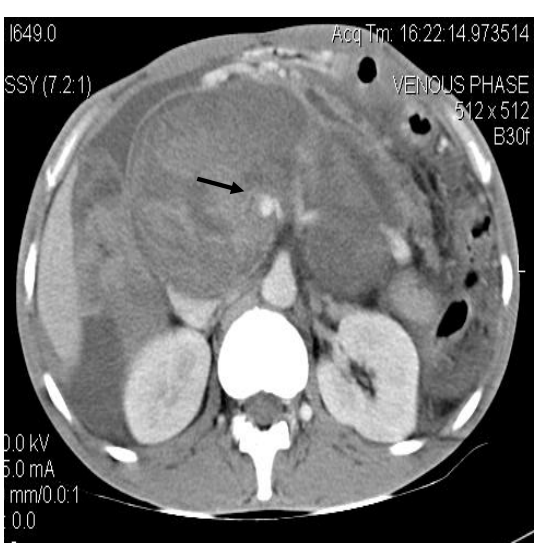

(c)

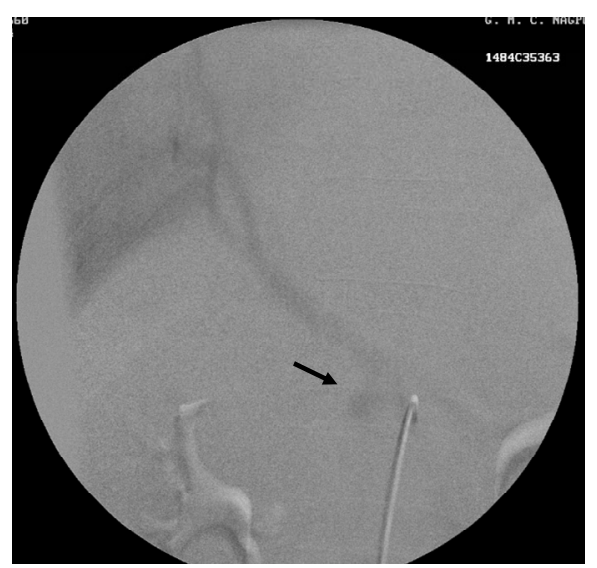

(d)

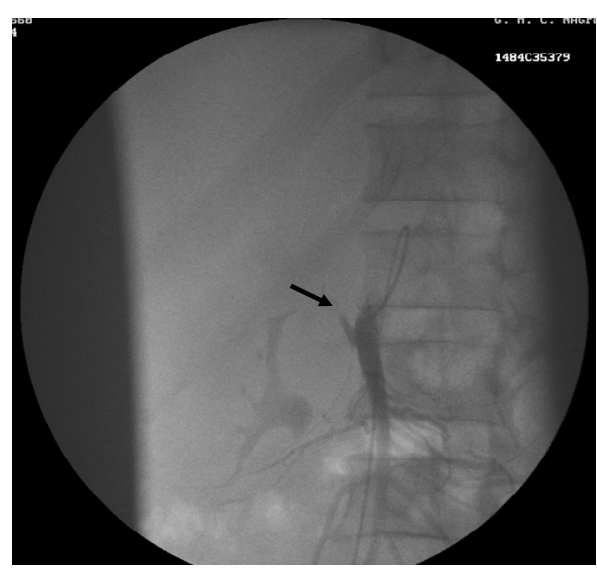

(e)

Figure 1. (a) Contrast enhanced CT scan showing the peripancreatic hypodense collection; (b) Repeat CT scan after 15 days showing the hyperdense nature of the same peripancreatic collection suggesting hemorrhage possibly due to vascular injury; (c) showing the pseudo aneurysm arising from the hepatic artery which itself had aberrant origin from the superior mesenteric artery; (d) Preembolisation DSA angiography image showing the pseudoaneurysm at the origin of the hepatic artery; (e) Post embolization cine Angiography image showing the embolization coils in the hepatic artery with non filling of the pseudo aneurysm and the hepatic artery itself, but proper contrast filling the superior mesenteric artery. 


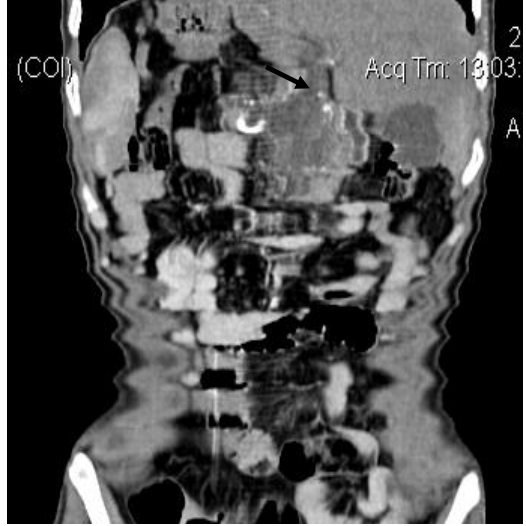

(a)

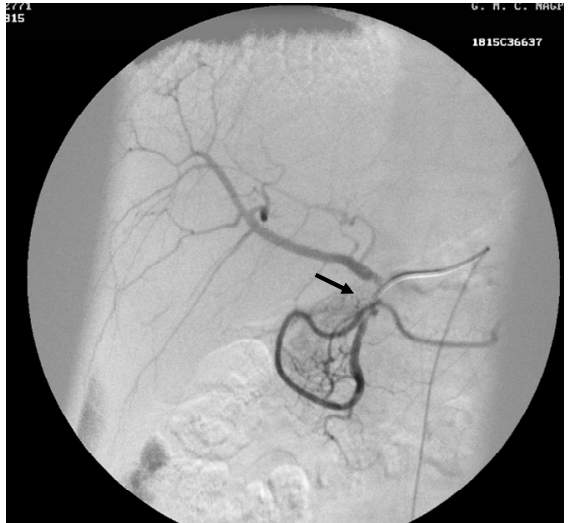

(b)

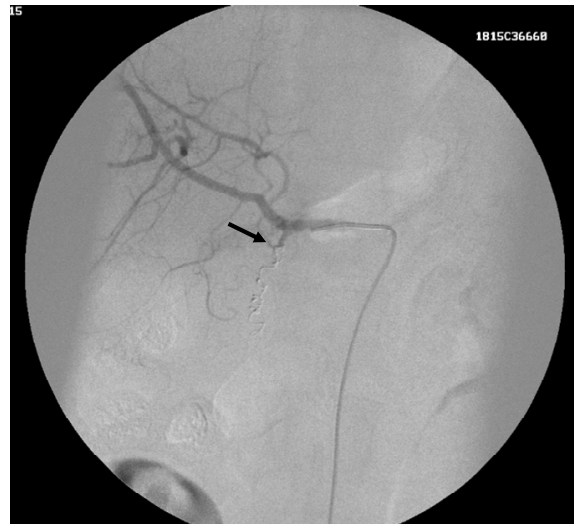

(c)

Figure 2. (a) coronal reformatted Contrast enhanced CT image showing the small pseudoaneurysm arising from the gastro-duodenal artery; (b) DSA angiography image showing the small pseudoaneurysm arising from the gastro duodenal artery; (c) post embolization DSA angiography image showing the coil embolization of the gastroduodenal artery with exclusion of the pseudoaneurysm from the circulation.

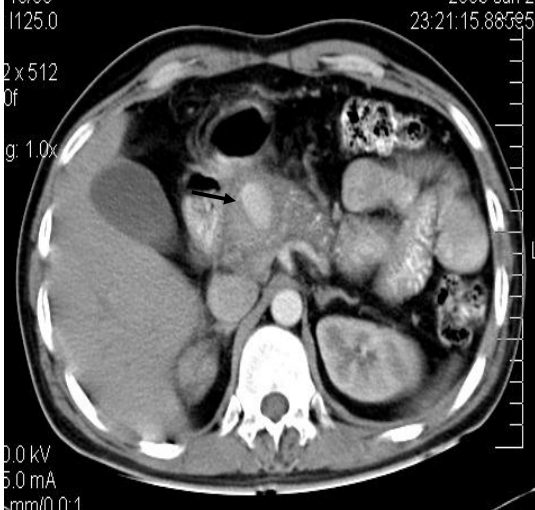

(a)

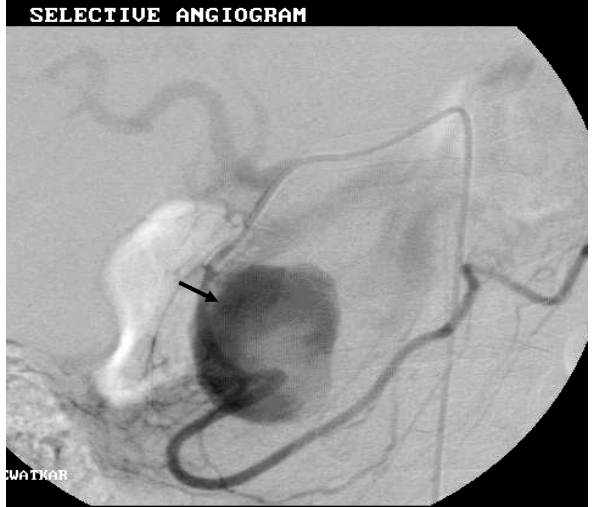

(b)

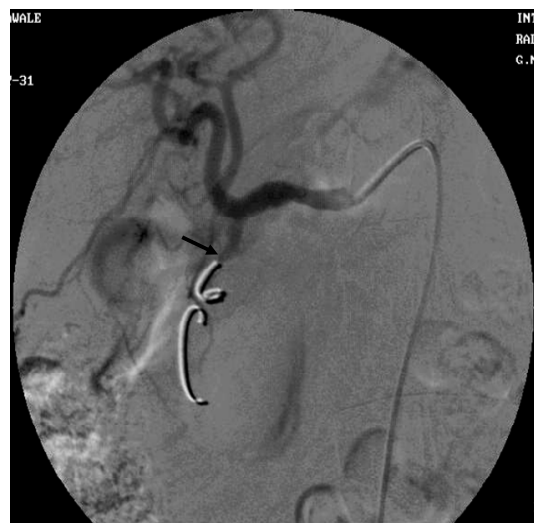

(c)

Figure 3. (a) Contrast enhanced CT scan showing large pseudo aneurysm arising from the gastro duodenal artery along with bulky and heterogeneous pancreas; (b) DSA angiography image showing the large pseudo aneurysm; (c) Post embolization DSA Angiography image showing the coils embolization and resultant non-filling of the gastro duodenal artery.

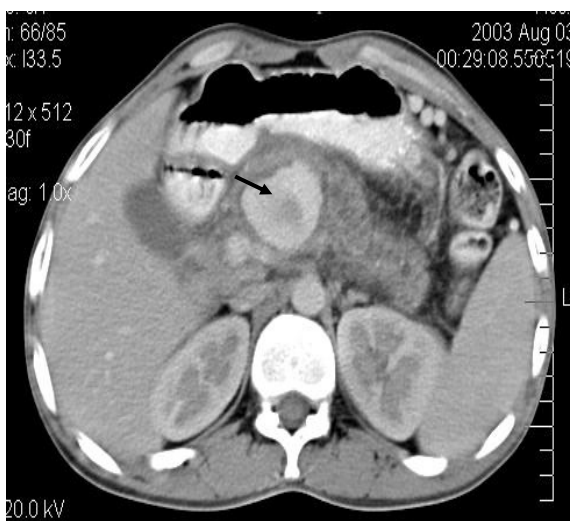

(a)

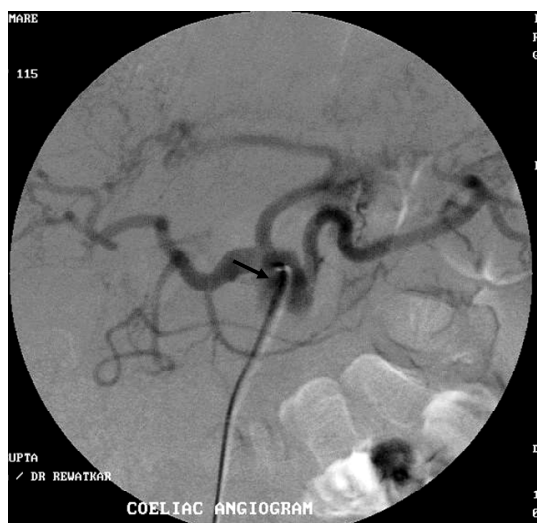

(b)

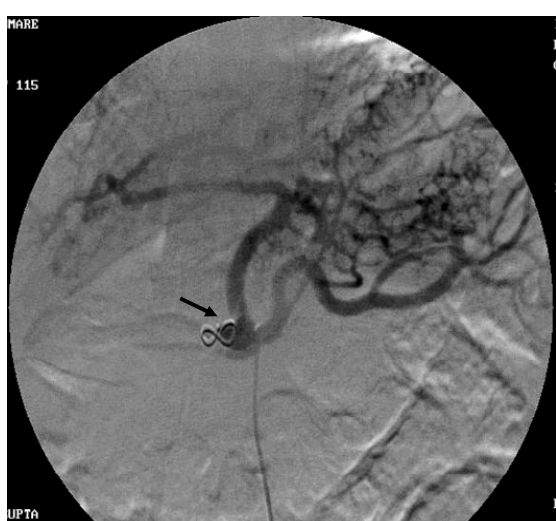

(c)

Figure 4. (a) Contrast enhanced CT scan showing the large pseudoaneurysm arising from the common hepatic artery with features of pancreatitis; (b) Selective Celiac trunk DSA angiography image showing the pseudoaneurysm at the proximal part of the common hepatic artery; (c) Post embolization DSA angiography image showing the non-filling of the common hepatic artery and embolization coils in situ. 


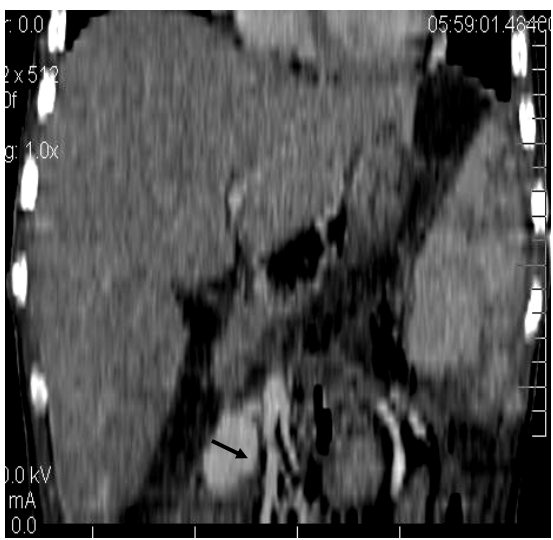

(a)

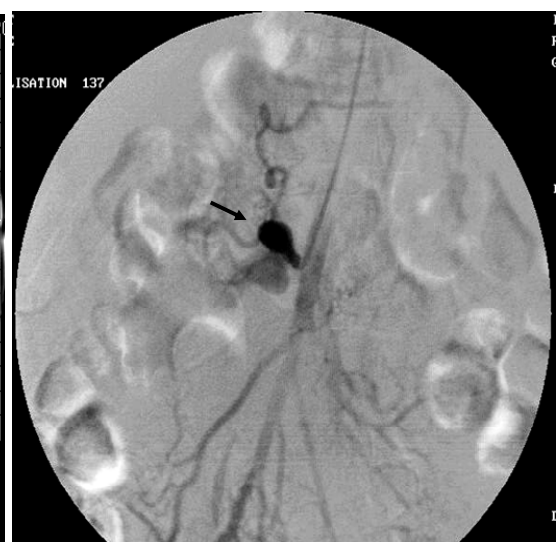

(b)

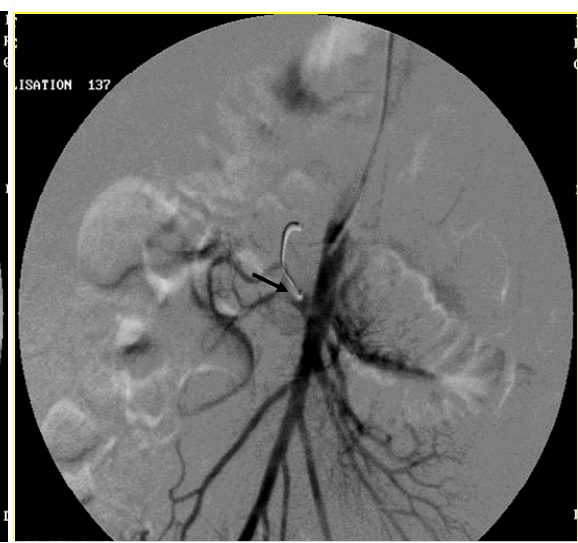

(c)

Figure 5. (a) Contrast enhanced coronal reformatted CT scan image showing the large pseudo aneurysm arising from the inferior pancreatico-duodenal artery; (b) Selective DSA angiography image of the superior mesenteric artery showing the pseudo aneurysm; (c) Post embolization Check angiography image showing the coil embolization with non contrast filling of the pseudo aneurysm.

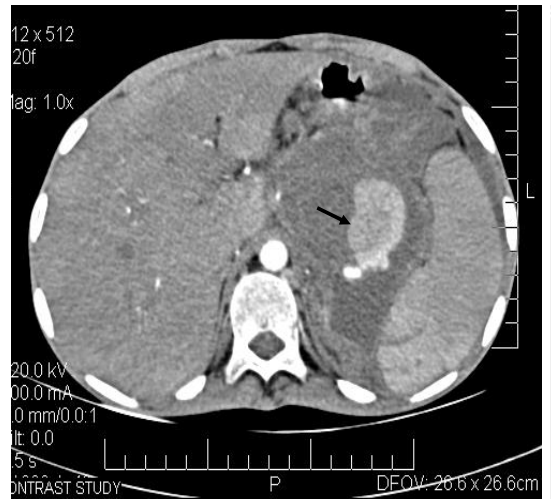

(a)

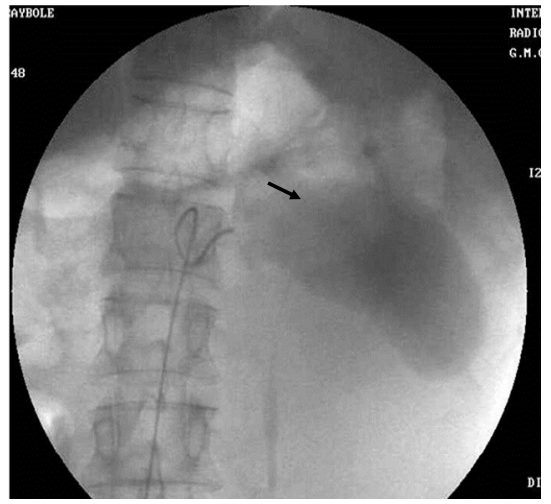

(b)

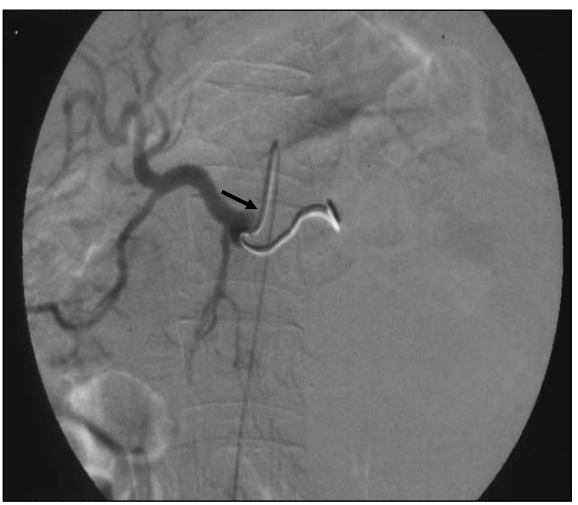

(c)

Figure 6. (a) Post contrast CT scan image showing the pseudoaneurysm arising from the splenic artery; (b) Pre embolization selective splenic arterial DSA angiography image showing pseudoaneurysm; (c) Post embolisation DSA image showing the coils inside the splenic artery with its resultant embolization.

analgesics. Each of the patients had been on follow up for at least 6 months, except 2 of them, with no new complaints in any of them. The procedure related mortality was none.

\section{Discussion}

A Pseudoaneurysm, also termed a false aneurysm, is a leakage of arterial blood from an artery into the surrounding tissue with a persistent communication between the originating artery and the resultant adjacent cavity, and contained by overlying media, adventitia or by soft tissue structures surrounding the injured vessels.

The etiology of these pseudoaneurysms may be varied, from inflammation, trauma to iatrogenic causes such as surgical procedures [3]. There is increased prevalence of pseudoaneurysms due to marked increase in the rate of surgical and angiographic procedures [4].

Sometimes these pseudoaneurysms may be asymp- tomatic and detected incidentally [5]. The symptoms due to pseudoaneurysms may be due to mass effect on the adjacent neuro-vascular structures causing compromise of their function or due to life threatening haemorrhage from their rupture [6-8]. As per literature, the rupture of splenic artery pseudoaneurysm has a mortality rate approaching 100\% [9]. The various possible sites of pseudoaneurysm rupture include gut, biliary tree, thoracic, peritoneal, pelvic and retroperitoneal spaces [10], which may manifest as sentinel bleed from the drain, hemetemesis, malena, splenic rupture or subcapsular hepatic hematoma [8,11].

The diagnosis of pseudoaneurysms has been revolutionised by varying imaging modalities like duplex Doppler ultrasonography (US), magnetic resonance (MR) angiography and helical computed tomography (CT) angiography [6,12] although the conventional angiography remains the gold standard of reference [13]. 


\section{Imaging Features}

Ultrasonography(US) — gray scale US demonstrates a hypoechoic cystic structure adjacent to supplying artery $[4,14,15]$. The various characteristics of the pseudoaneurysms that are to be assessed by US are the size of the sac, the connection of the sac to the artery, and the length and width of the neck [16]. Doppler US characteristically shows the "yin-yang sign" which refers to the to and fro blood movement from the neck into the pseudoaneurysmal sac in the systolic and diastolic phases respectively [11]. Advantages of the US are portable, readily available, inexpensive, fast, no ionising radiation or renal toxic contrast agent used and noninvasive [17-19]. The usefulness of US in the evaluation of visceral arteries is limited [20]. The other disadvantages of the US include operator dependency, and difficulty in trauma patients [12].

CT Angiography-unenhanced CT scan may show low attenuation rounded structure arising from the donor artery, with surrounding high attenuation rim in cases of rupture. Contrast enhanced CT scan may show a contrast material filled sac [21]. The advantages of CT angiography are shorter acquisition time, less operator dependency, generation of post-processed $3 \mathrm{D}$ angiography images to plan for surgery, detection of other organ injury simultaneously. The limitations of CT angiography include inferior spatial resolution as compared to conventional angiography and need for relatively more contrast agent and no therapeutic option [22].

MR Angiography - many new advances in the field of non contrast and contrast enhanced MRI Angiography have made the detection of subtle lesions possible. The advantages of MR Angiography include no iodinated contrast agent or ionising radiations $[23,24]$, thus making it the investigation of choice in patients of renal failure and contrast allergy. The limitations of MRI Angiography include time consumption, not suitable in trauma patients and artifacts due to multiple causes.

Conventional Angiography-it remains the gold standard of reference for the diagnosis of pseudoaneurysm even today [22,24,25]. The advantages of conventional angiography include real time hemodynamic assessment of the vascular bed, exact measurement of the size of the sac and neck of the pseudoaneurysm [26] and therapeutic intervention [12,22]. The disadvantages of conventional angiography include exposure to ionising radiations, procedure related complications, and inability to access the correct size of pseudoaneurysmal sac containing thrombus.

Thus, all these imaging modalities are in fact complementary to each other in the final diagnostic and therapeutic procedures.

The risk of spontaneous rupture of extraorganic visceral pseudo aneurysm is very high regardless of their size and the mortality rate for such ruptures in morbid postsurgical patients approaches to about 100\% [8,9,11]. Hence, definitive treatment should be administered in all such cases $[9,19]$. The traditional treatment of choice was surgical repair which was invasive and had higher morbidity and mortality rates $[7,16,27]$. In recent times various other treatment options have become available as a better alternative to surgery.

Endo-Vascular Management- the treatment option depends upon two important factors 1) size of pseudoaneurysmal neck and 2) expendability of the donor artery. If the donor artery is expendable, then the collateral supply is accessed. In case of no collateral supply, only proximal embolisaton of the parent artery is considered, but if the collateral supply is good, then both proximal and distal embolization of the parent artery with respect to the pseudoaneurysm is needed. If the artery is inexpendable, the neck size of the pseudoaneurysm is evaluated. In cases of narrow neck, embolization of the pseudoaneurysmal sac itself is considered, but if the neck is wide, then stent graft placement can be considered. In cases of the involvement of branches of the celiac trunk and superior mesenteric arteries especially due to pancreatitis, there is good collateral circulation $[11,26]$ and so the "Sandwich technique" of embolization is used in which the embolization is done distally and then proximally with respect to the site of the pseudoaneurysm to prevent backflow from the collateral circulation [26]. The preferred embolization agent of choice is catheter directed endovascular delivery of microcoils. Embolization microcoils fall into two categories Non-Detachable and Detachable. The non-detachable microcoils are those which resume their shape immediately after deployment from the catheter, made of either stainless steel or platinum, and may have incorporated polyester fibres in them to make them more thrombogenic. The development of the soft complex helical microcoils which assume the shape of the pseudoaneurysmal sac has made it possible the reduction in the number of microcoils used during the procedure. The platinum microcoils are softer and hence initial placement of the stainless steel microcoils is necessary to act as scaffolding. The detachable microcoils are those which are held to the pusher guide wire by either a mechanical or electrochemically dissolvable connection, which is then released to deploy the coil, which allows accurate deployment of the coil and repositioning of the coil before final deployment. The possible disadvantages of using coil embolization are potential for recanalisation of the embolised sac if the microcoils are not packed tightly. To avoid this, the microcoils may be supplemented with glue or thrombin [26]. If the neck of the pseudoaneurysmal sac is wide, then microcoils may be inserted inside the sac itself with proper precautions to prevent distal embolization. Stent-graft placement re- 
quires a higher profile and a stiffer delivery system and hence the visceral pseudoaneurysms which are usually smaller in size and arise from tortuous donor arteries pose a challenge for this method. In cases of visceral pseudoaneurysms, the endovascular techniques supersede surgical management $[5,10]$ with possible complications including intraprocedural pseudoaneurysmal rupture [9] and recanalisation of the embolised vessel and delayed failure of the embolization, which is however very rare [9].

Surgery - the various surgical procedures include resection and bypass procedure, arterial ligation, and partial and total organ removal [28]. Surgery in preferred in the following cases 1) pseudo aneurysms causing mass effect in order to rapidly reduce it; 2) infected pseudoaneurysms; 3) cases in which endovascular management has failed [29]. But surgical mortality is approximately $16 \%$ for pseudoaneurysms in the head of the pancreas and $50 \%$ for lesions in the tail [30].

The other possible therapeutic options are US guided compression and US guided percutaneous thrombin injection, which is usually suited for superficially located pseudoaneurysms [29].

\section{Conclusion}

Vascular complications of pancreatitis need immediate and accurate diagnosis and prompt treatment. It can be suspected on plain and contrast CT scan of the patient complaining of another episode of severe abdominal pain after recovery from initial pain of pancreatitis. Conventional angiography is done for further anatomic characterization and therapeutic management such as embolization. Micro coil embolisation, is a minimally invasive technique which helps to exclude the pseudoaneurysm from the circulation and thus reduces the scope for massive life threatening internal hemorrhage, and is a better alternative to surgery.

\section{REFERENCES}

[1] J. W. Burke, S. J. Erickson, C. D. Kellum, C. J. Tegtmeyer, B. R. J. Williamson and M. F. Hansen, "Pseudoaneurysms Complicating Pancreatitis: Detection with CT," Radiology, Vol. 161, No. 2, 1986, pp. 447-450.

[2] R. T. Woodburne and L. L. Olsen, "The Arteries of the Pancreas,” The Anatomical Record, Vol. 111, No. 2, 1951, pp. 255-270. doi:10.1002/ar.1091110209

[3] L. B. Schwartz, E. T. Clark and B. L. Gewertz, "Anastomotic and Other Pseudoaneurysms,” In: R. B. Rutherford, Ed., Vascular Surgery, 5th Edition, Saunders, Philadelphia, 2000, pp. 752-763.

[4] P. J. Bromley, T. Clark, I. H. Weir and C. V. Zwirewich, "Radiologic Diagnosis and Management of Uterine Artery Pseudoaneurysm: Case Report,” Canadian Associa- tion of Radiologists Journal, Vol. 48, No. 2, 1997, pp. 119-122.

[5] V. G. McDermott, R. Shlansky-Goldberg and C. Cope, "Endovascular Management of Splenic Artery Aneurysms and Pseudoaneurysms,” CardioVascular and Interventional Radiology, Vol. 17, No. 4, 1994, pp. 179-184. doi:10.1007/BF00571531

[6] A. Ahmed, S. L. Samuels, E. B. Keeffe and R. C. Cheung, "Delayed Fatal Hemorrhage from Pseudoaneurysm of the Hepatic Artery after Percutaneous Liver Biopsy,” The American Journal of Gastroenterology, Vol. 96, No. 1, 2001, pp. 233-237. doi:10.1111/j.1572-0241.2001.03482.x

[7] L. La Perna, J. W. Olin, D. Goines, M. B. Childs and K. Ouriel, "Ultrasound-Guided Thrombin Injection for the Treatment of Postcatheterization Pseudoaneurysms," Circulation, Vol. 102, No. 19, 2000, pp. 2391-2395. doi:10.1161/01.CIR.102.19.2391

[8] A. Okuno, M. Miyazaki, H. Ito, et al., "Nonsurgical Management of Ruptured Pseudoaneurysm in Patients with Hepatobiliary Pancreatic Diseases," The American Journal of Gastroenterology, Vol. 96, No. 4, 2001, pp. 10671071 doi:10.1111/j.1572-0241.2001.03691.x

[9] R. Guillon, J. M. Gracier, A. Abergel, et al., "Management of Splenic Artery Aneurysms and False Aneurysms with Endovascular Treatment in 12 Patients," CardioVascular and Interventional Radiology, Vol. 26, No. 3, 2003, pp. 256-260 doi:10.1007/s00270-003-1948-y

[10] K. Yamakado, A. Nakatsuka, N. Tanaka, K. Takano, K. Matsumura and K. Takeda, "Transcatheter Arterial Embolization of Ruptured Pseudoaneurysms with Microcoils and n-Butyl Cyanoacrylate,” Journal of Vascular and Interventional Radiology, Vol. 11, No. 1, 2000, pp. 66-72. doi:10.1016/S1051-0443(07)61284-6

[11] J. F. Polak, “The Peripheral Arteries,” In: C. M. Rumack, S. R. Wilson and J. W. Charboneau, Eds. Diagnostic Ultrasound, 2nd Edition, Mosby, St. Louis, 1998, pp. 921941.

[12] J. A. Soto, F. Munera, C. Morales, et al., "Focal Arterial Injuries of the Proximal Extremities: Helical CT Arteriography as the Initial Method of Diagnosis,” Radiology, Vol. 218, No. 1, 2001, pp. 188-194.

[13] A. E. Zimon, J. K. Hwang, D. L. Principe and R. O. Bahado-Singh, "Pseudoaneurysm of the Uterine Artery," $\mathrm{Ob}$ stetrics \& Gynecology, Vol. 94, No. 5, 1999, pp. 827-830. doi:10.1016/S0029-7844(99)00229-X

[14] J. D. Crossin, D. Muradali and S. R. Wilson, "US of Liver Transplants: Normal and Abnormal,” RadioGraphics, Vol. 23, No. 5, 2003, pp. 1093-1114. doi:10.1148/rg.235035031

[15] J. H. Kwon and G. S. Kim, “Obstetric Iatrogenic Arterial Injuries of the Uterus: Diagnosis with US and Treatment with Transcatheter Arterial Embolization,” RadioGraphics, Vol. 22, No. 1, 2002, pp. 35-46.

[16] K. Kruger, M. Zahringer, F. D. Sohngen, et al., "Femoral Pseudoaneurysms: Management with Percutaneous Thrombin Injections-Success Rates and Effects on Systemic Coagulation,” Radiology, Vol. 226, No. 2, 2003, pp. 452 458. doi:10.1148/radiol.2262012107 
[17] G. Brancatelli, S. Katyal, M. P. Federle and P. Fontes, "Three-Dimensional Multislice Helical Computed Tomography with the Volume Rendering Technique in the Detection of Vascular Complications after Liver Transplantation," Transplantation, Vol. 73, No. 2, 2002, pp. 237242. doi:10.1097/00007890-200201270-00015

[18] D. J. Tessier, W. M. Stone, R. J. Fowl, et al., "Clinical Features and Management of Splenic Artery Pseudoaneurysm: Case Series and Cumulative Review of Literature,” Journal of Vascular Surgery, Vol. 38, No. 5, 2003, pp. 969-974. doi:10.1016/S0741-5214(03)00710-9

[19] D. S. Katz and M. Hon, "CT Angiography of the Lower Extremities and Aortoiliac System with a Multi-Detector Row Helical CT Scanner: Promise of New Opportunities Fulfilled,” Radiology, Vol. 221, No. 1, 2001, pp. 7-10. doi:10.1148/radiol.2211011087

[20] S. Katyal, J. H. Oliver 3rd, D. G. Buck and M. P. Federle, "Detection of Vascular Complications after Liver Transplantation: Early Experience in Multislice CT Angiography with Volume Rendering," American Journal of Roentgenology, Vol. 175, No. 6, 2000, pp. 1735-1739.

[21] B. L. Knisely, L. A. Mastey, J. Collins and J. E. Kuhlman, "Imaging of Cardiac Transplantation Complications," RadioGraphics, Vol. 19, No. 2, 1999, pp. 321-341.

[22] F. Munera, J. A. Soto, D. Palacio, S. M. Velez and E. Medina, "Diagnosis of Arterial Injuries Caused by Penetrating Trauma to the Neck: Comparison of Helical CT Angiography and Conventional Angiography,” Radiology, Vol. 216, No. 2, 2000, pp. 356-362.

[23] J. F. Glockner, “Three-Dimensional Gadolinium-Enhanced MR Angiography: Applications for Abdominal Imaging,” RadioGraphics, Vol. 21, No. 2, 2001, pp. 357-370.
[24] M. V. Nastri, L. P. Baptista, R. H. Baroni, et al., "Gadolinium-Enhanced Three-Dimensional MR Angiography of Takayasu Arteritis,” RadioGraphics, Vol. 24, No. 3, 2004, pp. 773-786. doi:10.1148/rg.243035096

[25] A. R. Busquets, J. A. Acosta, E. Colon, K. V. Alejandro and P. Rodriguez, "Helical Computed Tomographic Angiography for the Diagnosis of Traumatic Arterial Injuries of the Extremities," The Journal of Trauma, Vol. 56, No. 3, 2004, pp. 625-628. doi:10.1097/01.TA.0000053546.28739.CF

[26] M. A. Arata and C. Cope, "Principles Used in the Management of Visceral Aneurysms,” Techniques in Vascular and Interventional Radiology, Vol. 3, No. 3, 2000, pp. 124 129. doi:10.1053/tvir.2000.9147

[27] C. Thalhammer, A. S. Kirchherr, F. Uhlich, J. Waigand and C. M. Gross, "Postcatheterization Pseudoaneurysm and Arteriovenous fistulas: Repair with Percutaneous Implantation of Endovascular Covered Stents," Radiology, Vol. 214, No. 1, 2000, pp. 127-131.

[28] H. Bergert, I. Hinterseher, S. Kersting, J. Leonhardt, A. Bloomenthal and H. D. Saeger, "Management and Outcome of Hemorrhage Due to Arterial Pseudoaneurysms in Pancreatitis,” Surgery, Vol. 137, No. 3, 2005, pp. 323328. doi:10.1016/j.surg.2004.10.009

[29] R. Morgan and A. Belli, "Current Treatment Methods for Postcatheterization Pseudoaneurysms," Journal of Vascular and Interventional Radiology, Vol. 14, No. 6, 2003, pp. 697-710. doi:10.1097/01.RVI.0000071089.76348.6A

[30] C. D. Long, K. R. Bakshi, M. B. Kahn and A. B. Roberts, "Giant Splenic Artery Aneurysm," Annals of Vascular Surgery, Vol. 7, No. 5, 1993, pp. 474-478. doi:10.1007/BF02002133 